\title{
Photoelectric detection technology of laser seeker signals
}

\author{
ZHU Likun ${ }^{1,2}$, JIA Fangxiu ${ }^{1, *}$, JIANG Xiaodong ${ }^{1}$, and LI Xinglong ${ }^{3}$ \\ 1. Minsterial Key Laboratory of ZNDY, Nanjing University of Science and Technology, Nanjing 210094, China; \\ 2. CETHIK GROUP CO., LTD, Hangzhou 310012, China; \\ 3. Institute of Chemical Materials, China Academy of Engineering Physics, Mianyang 621900, China
}

\begin{abstract}
The measurement of the rolling angle of the projectile is one of the key technologies for the terminal correction projectile. To improve the resolution accuracy of the rolling angle in the laser seeker weapon system, the imaging model of the detector, calculation model of the position and the signal-to-noise ratio (SNR) model of the circuit are built to derive both the correlation between the resolution error of the rolling angle and the spot position, and the relation between the position resolution error and the SNR. Then the influence of each parameter on the SNR is analyzed at large, and the parameters of the circuit are determined. Meanwhile, the SNR and noise voltage of the circuit are calculated according to the SNR model and the decay model of the laser energy. Finally, the actual photoelectric detection circuit is built, whose SNR is measured to be up to $53 \mathrm{~dB}$. It can fully meet the requirement of $0.5^{\circ}$ for the resolution error of the rolling angle, thereby realizing the analysis of critical technology for photoelectric detection of laser seeker signals.
\end{abstract}

Keywords: laser seeker, rolling angle, error of position, signal-tonoise ratio (SNR), photoelectric detection.

DOI: $10.21629 / J S E E .2019 .06 .02$

\section{Introduction}

The laser semi-active search guide weapon system (LSSGWS) is featured with high guidance accuracy, strong antijamming ability, low cost, simple structure and usableness, etc. It is extensively applied to the weapons and most frequently used as photoelectric precision guided weapon $[1-5]$. The working principle of LSSGWS is as follows. When the target is irradiated with the encoded laser beam on the airborne or ground surface, the laser seeker can receive the echo information of the diffuse reflection, and the information is transmitted to the guidance system to track the target. At the same time, the deviation signal is transmitted to the projectile control system to guide the projec-

Manuscript received June 26, 2018

*Corresponding author.

This work was supported by the National Natural Science Foundation of China (61201391). tile to the target.

The LSSGWS working function mainly depends on the laser seeker head on the front end of the weapon [6-8]. According to the correction strategy, the projectile obtains the steering instruction of the actuator. When the projectile rolls to a specific angle, the actuator is started up, which produces the controlling force to correct the trajectory [911]. Thus, the accuracy of measurement of the rolling angle has a direct bearing upon the correct accuracy [12].

When the laser with repeated frequency of $10-20 \mathrm{~Hz}$ is used for guidance, the detector mostly adopts the optical position sensor with quick velocity response and will output the nanosecond impulse signal [13]. During the actual appliance, the performance of the detecting instrument will be influenced and limited by the noise and interference [14-17]. In the process of laser guidance, the optical signal is relatively weak when the target has low reflectivity or a large transmission range. While the primary function of the photoelectric detection circuit is to eliminate the influence of noise and interference on the detector and raise the signal-to-noise ratio (SNR) of its circuit as far as possible, which can be obtained to a higher guidance accuracy.

Related scholars analyzed the influencing factors of noise in the photoelectric detection circuit and the problems requiring attention in the design of the detection circuit [18-24]. Liang et al. analyzed the influencing factors for position accuracy of the four-quadrant detector [6]. Jiang et al. tested and analyzed the equivalent noise of the position sensitive detector (PSD) measuring circuit, obtaining a series of simulation results [25]. However, the existing analyses on the photoelectric detection circuit are all based on the quantitative analysis, noise model analysis and analog simulation analysis instead of making specific analyses on the characteristics of the laser homing head signal. Thus, the relation between the position resolution accuracy and the resolution error of the rolling angle on the imaging face of the photosensor is firstly analyzed according to the trajectory information and the characteristics of 
the correction projectile based on the laser homing head, deducing the resolution error relation of error determination position for the rolling angle. Then the SNR model is analyzed by the relation between the position error and the SNR. Finally the factors influencing the circuit SNR are analyzed. According to the characteristics of the laser homing head signal, a low noise photoelectric detection circuit is built. In addition, the related measurement is carried out to verify whether the designed circuit can deliver the error precision of rolling angle resolution.

\section{Resolution error of rolling angle and spot position}

The LSSGWS of the schematic diagram of the working principle is illustrated in Fig. 1.

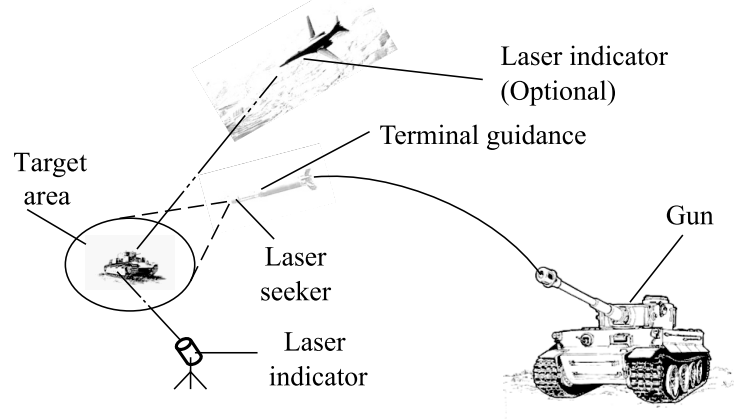

Fig. 1 LSSGWS of the schematic diagram of the working principle

In order to illustrate the relationship between the imaging plane of the projectile and the real target point on the ground, the imaging model is established as Fig. 2.

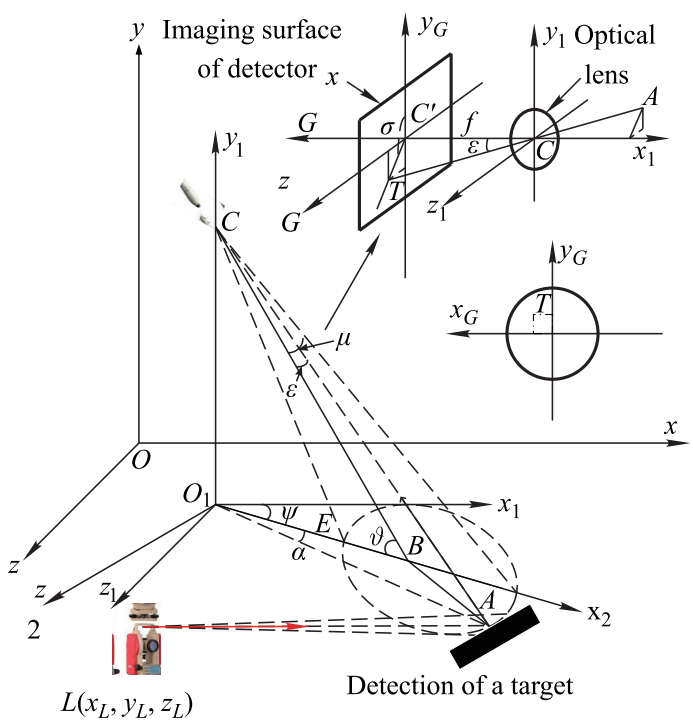

Fig. 2 Schematic diagram of the imaging model of photoelectric position sensor
In the figure above, the $O-x y z$ is the ground coordinate system, and the coordinate system $O_{1}-x_{1} y_{1} z_{1}$ is obtained by transferring from the ground coordinate system to the projection point $O_{1}$ of the projectile on the ground. Definition of the projectile coordinate system $O_{1}-x_{2} y_{2} z_{2}$ : The coordinate system $O_{1}-x_{1} y_{1} z_{1}$ is rotated $\psi$ clockwise around $O_{1} y_{1}$ axis, and $\Psi$ is the yaw angle. Point $C$ is the position of the detector lens on the projectile; Point $B$ is the intersection point of the projectile shaft axis or the optical axis and the axis $x_{2}$ of the projectile; Point $A$ is the location of the target point; $\vartheta$ is the pitch angle of the projectile. An aspect angle of the projectile $\varepsilon$ is defined as the angle between the projectile shaft axis and the connection between the projectile and the target, which represents the degree of deviation from the target.

At time $t, T\left(y_{g}, z_{g}\right)$ is the projection point of the target point measured on the imaging surface of the detector; $C\left(x_{0}, y_{0}, z_{0}\right)$ is the point of the ground system coordinates; $\theta$ is the pitch angle, $\psi$ is the yaw angle, $\gamma$ is the roll angle. The lens imaging relationship between the light spot coordinate $T$ on the imaging surface of the sensor and the imaging point $A$ in the missile body coordinate system $O_{1}^{\prime}-x_{1} y_{1} z_{1}$ of the projectile, is shown in Fig. 2. Ignoring the installation error between the sensor and the lens, the light spot coordinate $T$ on the imaging surface of the sensor is transformed into other coordinates. The specific conversion process is as follows.

Firstly, the coordinates of the imaging point $T$ in the coordinate system $O_{1}-x_{1} y_{1} z_{1}$ are calculated, and this process is used to realize by the coordinate transformation matrix.

Secondly, it is known that the position of the projectile $C$ is $\left(0, y_{0}, 0\right)$ under the coordinate system $O_{1}-x_{1} y_{1} z_{1}$. Because two points determine a straight line, the intersection point of the straight line $T C$ and the plane $x_{1} O_{1} z_{1}$ is the position coordinates of the target point $A$, shown as Fig. 2.

Thirdly, the point $A$ is converted to the ground coordinate system $O_{1}-x y z$. After the above derivation, the conversion of the target point and the light spot can be linked.

From the above analysis, the calculation accuracy of the roll angle of the projectile is related to the seven parameters of $x_{0}, y_{0}, z_{0}, \vartheta, \psi, y_{G}, z_{G}$. The error of the roll angle is $\Delta \gamma$, and the seven errors are $\Delta x_{0}, \Delta y_{0}, \Delta z_{0}, \Delta \vartheta, \Delta \psi, \Delta y_{G}, \Delta z_{G}$. The error can be theoretically obtained as below:

$$
\begin{gathered}
\Delta \gamma=\left|\frac{\partial \gamma}{\partial x_{0}}\right| \Delta x_{0}+\left|\frac{\partial \gamma}{\partial y_{0}}\right| \Delta y_{0}+\left|\frac{\partial \gamma}{\partial z_{0}}\right| \Delta z_{0}+\left|\frac{\partial \gamma}{\partial \vartheta}\right| \Delta \vartheta+ \\
\left|\frac{\partial \gamma}{\partial \psi}\right| \Delta \psi+\left|\frac{\partial \gamma}{\partial y_{G}}\right| \Delta y_{G}+\left|\frac{\partial \gamma}{\partial z_{G}}\right| \Delta z_{G}
\end{gathered}
$$


with

$$
\left\{\begin{array}{l}
\frac{\partial \gamma}{\partial x_{0}}=-\frac{\sin \vartheta \cdot x_{1}+b_{1} \cdot \cos \vartheta \cdot \cos \psi}{d_{0}} \\
\frac{\partial \gamma}{\partial y_{0}}=-\frac{\cos \vartheta \cdot x_{1}+b_{1} \cdot \sin \vartheta \cdot \cos \psi}{d_{0}} \\
\frac{\partial \gamma}{\partial z_{0}}=\frac{b_{1} \cdot \sin \psi}{d_{0}} \\
\frac{\partial \gamma}{\partial \vartheta}=-\frac{d_{1} \cdot x_{1}+b_{1} \cdot \sigma}{d_{0}} \cdot \lambda \\
\frac{\partial \gamma}{\partial \psi}=-\frac{b_{1} \cdot \tan \varepsilon \cdot d_{2}}{d_{0}} \cdot \kappa \\
\frac{\partial \gamma}{\partial y_{G}}=-\frac{b_{1} \cdot f \cdot y_{G}}{\left(y_{G}^{2}+z_{G}^{2}\right)^{3 / 2} \cdot d_{0}}+\frac{z_{G}}{y_{G}^{2}+z_{G}^{2}} \\
\frac{\partial \gamma}{\partial z_{G}}=-\frac{b_{1} \cdot f \cdot z_{G}}{\left(y_{G}^{2}+z_{G}^{2}\right)^{3 / 2} \cdot d_{0}}-\frac{y_{G}}{y_{G}^{2}+z_{G}^{2}}
\end{array}\right.
$$

and

$$
\left\{\begin{aligned}
\phi_{1}= & \arctan \left(\tan \vartheta \cdot \sqrt{1+(\tan \psi)^{2}}\right) \\
\phi_{2}= & \arctan \left(\frac{\tan \psi}{\sqrt{1+(\tan \vartheta)^{2}+(\tan \vartheta)^{2}(\tan \psi)^{2}}}\right) \\
b_{1}= & -\Delta x \cdot \sin \phi_{1}+\Delta y \cdot \cos \phi_{1} \\
d_{0}= & x_{1}^{2} \cdot \tan \varepsilon \sqrt{1-b_{1}^{2} /\left(x_{1} \cdot \tan \varepsilon\right)^{2}} \\
d_{1}= & -\Delta x \cdot \cos \phi_{1}-y_{0} \cdot \sin \phi_{1} \\
d_{2}= & -\Delta x \cdot \cos \phi_{1} \cdot \sin \phi_{2}-\Delta y \cdot \sin \phi_{1} \cdot \sin \phi_{2}+ \\
& \Delta z \cdot \cos \phi_{2} \\
x_{1}= & \Delta x \cdot \cos \phi_{1} \cdot \cos \phi_{2}+\Delta y \cdot \sin \phi_{1} \cdot \cos \phi_{2}+ \\
\lambda= & \frac{\Delta z \cdot \sin \phi_{2}}{\sqrt{1+(\tan \psi)^{2}}} \\
& {\left[(\cos \vartheta)^{2}+(\cos \vartheta)^{2}(\tan \vartheta)^{2}+\right.} \\
\kappa= & \frac{(\tan \psi)^{2}}{(\tan \vartheta)^{2} \sqrt{1+(\tan \vartheta)^{2}+(\tan \vartheta)^{2}(\tan \psi)^{2}}} \\
\sigma= & -\Delta x \cdot \sin \phi_{1} \cdot \cos \phi_{2}-\Delta y \cdot \cos \phi_{1} \cdot \cos \phi_{2}
\end{aligned}\right.
$$

It is easy to get the error model of the rolling angle solution from above. To analyze the influence of precision of the rolling angle solution of the spot location error $\Delta y_{G}$ and $\Delta z_{G}$ on the sensor imaging surface, it is assumed that the nominal value of the other variables is constant and the error is zero, and the influence is discussed about the single error on the accuracy of the rolling angle solution.

The $120 \mathrm{~mm}$ laser semi-active correction projectile with a tail stabilizer is the study object. The initial velocity is $v=340 \mathrm{~m} / \mathrm{s}$ and the projectile mass is $m=13.45 \mathrm{~kg}$. In the simulation process, the range of $\left(\Delta y_{G}, \Delta z_{G}\right)$ is from $-0.1 \mathrm{~mm}$ to $0.1 \mathrm{~mm}$. After substituting them into the error model of the rolling angle solution, the total error of the rolling angle is illustrated as Fig. 3.

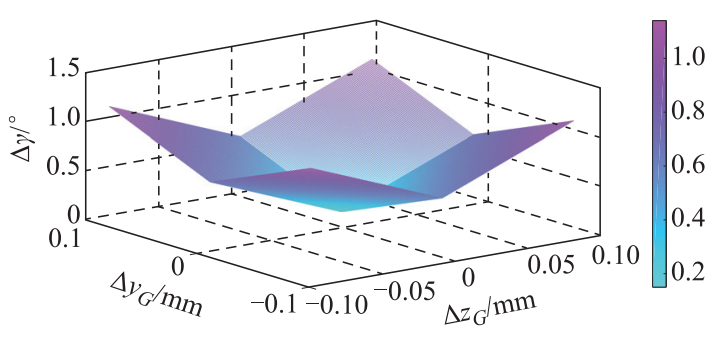

Fig. 3 Influence of the spot location error on the accuracy of the roll angle calculation

In the simulation process, because the starting light spot is assumed to fall on the positive and negative $45^{\circ}$ lines through the coordinates of the origin, the influence factor is equal. By simulation analysis, if the error of the spot position is only considered and the error of the rolling angle is controlled in the range of $0.5^{\circ}$, the position error should be controlled within the range of $50 \mu \mathrm{m}$.

\section{Design of photoelectric detection circuit}

In order to make the signal output of the photoelectric sensor easy to detect, the first thing how to convert the current signal from the sensor into a better measured voltage signal is considered. The signal of current source is converted into a voltage source, which can be achieved by using a voltage parallel negative feedback circuit [26-28].

Because the attenuation of the laser energy in the atmosphere is very serious, the guidance distance of the missile system is thousands of meters, and the output current signal of the sensor is so weak, it is easily disturbed by other various signals. Therefore, it is beneficial to increase the stability and anti-interference ability of the circuit by choosing reasonable electrical parameters according to the accuracy of modification.

\subsection{SNR and photocurrent}

Based on the above analysis, it can be seen that the schematic diagram of the constant current spot measuring circuit for two-dimensional (2-D) photoelectric sensor light spot mainly consists of the negative feedback circuit, the addition circuit, the subtraction circuit and the division circuit.

Because the illuminant is mostly the pulsed source light in the real application, the first-stage amplifier in the directcurrent measurement schematic diagram is added with the filter circuit. Besides, the resolving circuit of the spot point is realized through analog circuit. In addition, the number of bits and velocity of existing analog-to-digital converter (ADC) chip are enough to replace the functions of addition and division, which are prone to produce noise caused by temperature and environment.

Thus, the addition and division of the software are used to replace the function of analog circuit, as shown in Fig. 4 
in details. The electric current signal output by the photoelectric position sensor is processed in the amplifier in Fig. 4. Then it is directly sent to the data acquisition chip for data acquisition unit (DAU). After related operation, the position information of the spot point can be calculated.

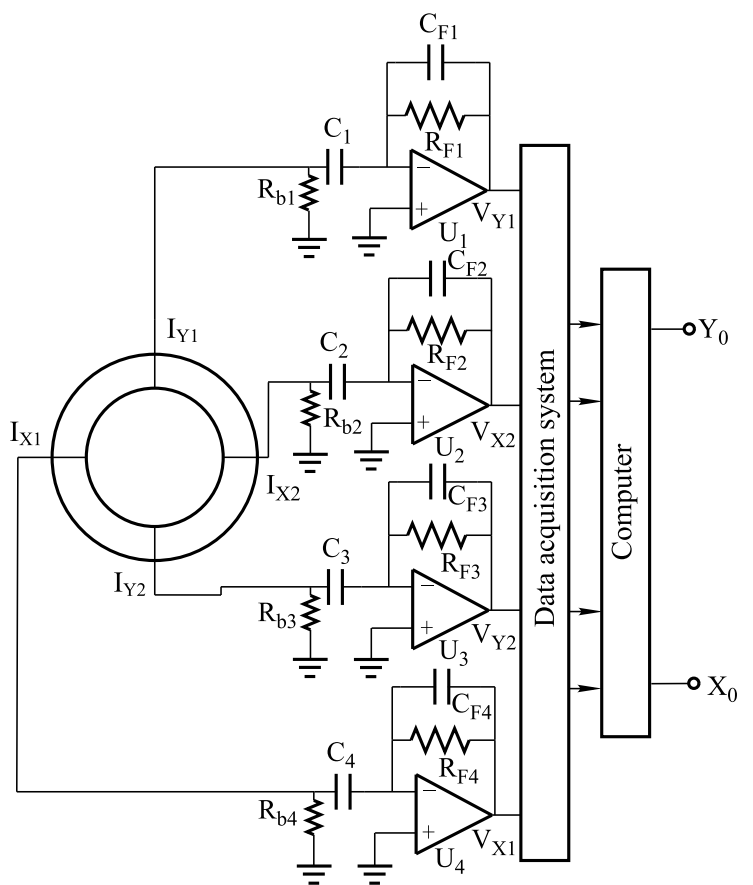

Fig. 4 Schematic diagram of signal processing circuit with software instead of the operator

From Fig. 4, it is known that the noise of the amplifier and other devices, the inequality of the resistance of resistor, the offset without elimination, and the temperature drift will lead to the error in the calculation of the coordinates. To derive the position resolution of the 2-D photoelectric position sensor, it is assumed that the incident light moves $\Delta x$ and $\Delta y$ on the imaging face, and then the photocurrent of the corresponding pole will change slightly. The change is recorded as $\Delta I$ shown in Fig. 5 .

Then (2) can be obtained.

$$
\begin{gathered}
x=\frac{L x}{2} \frac{\left(I_{X_{2}}+I_{Y_{1}}\right)-\left(I_{X_{1}}+I_{Y_{2}}\right)}{I_{X_{1}}+I_{X_{2}}+I_{Y_{1}}+I_{Y_{2}}}= \\
\frac{L x}{2} \frac{I_{1}-I_{2}}{I_{X_{1}}+I_{X_{2}}+I_{Y_{1}}+I_{Y_{2}}} \rightarrow \\
\Delta x=\frac{\Delta I_{X}-\Delta I_{Y}}{I_{O}} L x \\
y=\frac{L y}{2} \frac{\left(I_{X_{2}}+I_{Y_{2}}\right)-\left(I_{X_{1}}+I_{Y_{1}}\right)}{I_{X_{1}}+I_{X_{2}}+I_{Y_{1}}+I_{Y_{2}}}= \\
\frac{L y}{2} \frac{I_{3}-I_{4}}{I_{X_{1}}+I_{X_{2}}+I_{Y_{1}}+I_{Y_{2}}} \rightarrow \\
\Delta y=\frac{\Delta I_{X}+\Delta I_{Y}}{I_{O}} L y
\end{gathered}
$$

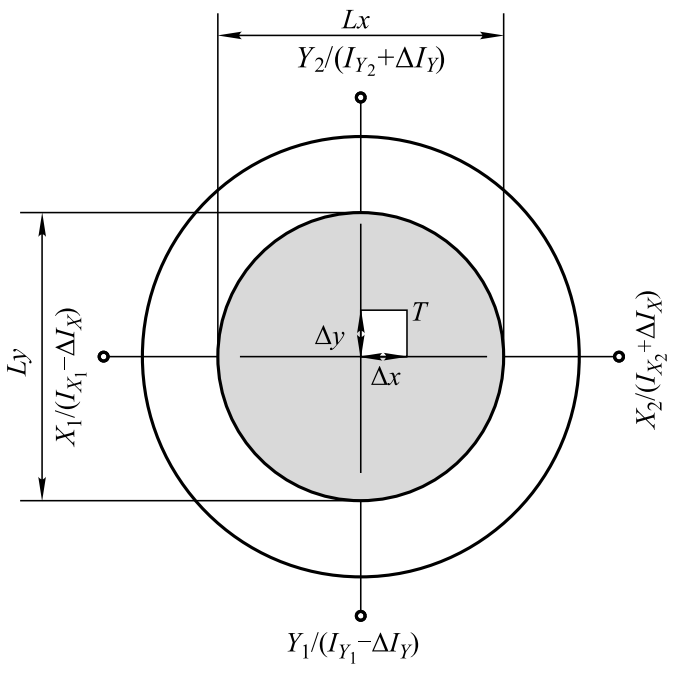

Fig. 5 Relationship between the displacement of the spot on the imaging surface and the photocurrent

and

$$
\begin{aligned}
& I_{1}=I_{X_{2}}+\Delta I_{X}+I_{Y_{1}}-\Delta I_{Y} \\
& I_{2}=I_{X_{1}}-\Delta I_{X}+I_{Y_{2}}+\Delta I_{Y} \\
& I_{3}=I_{X_{2}}+\Delta I_{X}+I_{Y_{2}}+\Delta I_{Y} \\
& I_{4}=I_{X_{1}}-\Delta I_{X}+I_{Y_{1}}-\Delta I_{Y}
\end{aligned}
$$

In the real application, when neglecting the machining error $(L=L x=L y)$, (9) can be obtained.

$$
\Delta x+\Delta y=2 \cdot \frac{\Delta I_{X}}{I_{o}} L
$$

According to (9), the resolution of the circuit is determined by the SNR of the detection circuit. The larger the photocurrent and the smaller the noise, the higher the resolution.

According to the correction accuracy, the location error of the spot can be easily controlled in the range of $50 \mu \mathrm{m}$. Assuming that the location error is $\Delta x=\Delta y= \pm 25 \mu \mathrm{m}$, the SNR of the circuit is up to $53 \mathrm{~dB}$ according to (9).

\subsection{Influence factors of noise}

According to Section 3.1, if reaching the desired positioning error, the SNR of the circuit is up to 480:1. The noise of the circuit is the main factor that affects the SNR of the detection circuit. Thus the next step is to deduce the SNR of the entire circuit from the noise model of the circuit.

The noise of an operational (OP) amplifier can be characterized by three equivalent noise sources, including current noise source with two spectral densities of $I_{n n}$ and $I_{n p}$ and a voltage noise source with a spectral density of $e_{n}$. These three noise sources are basically independent [29], and the circuit can be simplified as Fig. 6. In this picture, 
$I_{O}$ is photocurrent, $I_{D}$ is dark current, $R_{F}$ is feedback resistor, $C_{F}$ is parasitic capacitance of feedback resistor and $V o$ is output voltage.

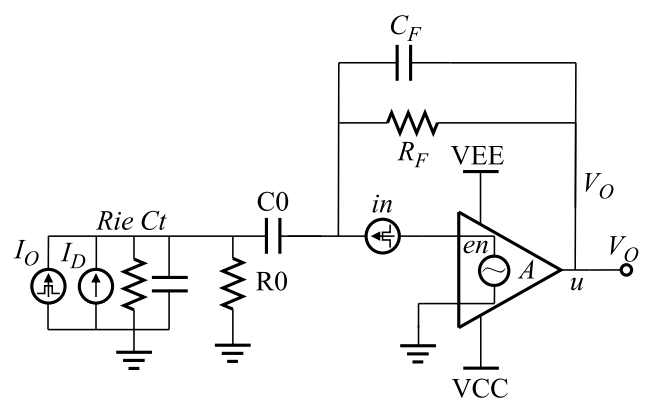

Fig. 6 Noise model circuit of 2-D photoelectric sensor

The noise root mean square (RMS) voltage of the circuit [25] is

$$
V n=\sqrt{V_{O}}
$$

with

$$
V_{O}=V s^{2}+V j^{2}+V e n^{2}+V_{R_{F}}^{2}+V i n^{2} .
$$

Among them (the unit is Volt/V and the same below): the bulk noise of photocurrent dark current is $V j=$ $R_{F} \cdot \sqrt{4 k T B / \text { Rie }} . B$ represents the bandwidth of the circuit, and $q$ represents the amount of electron charge, $q=1.6 \times 10^{-19} C$; the equivalent internal resistance thermal noise is $V j=R_{F} \cdot \sqrt{4 k T B / \text { Rie }} . T$ represents the absolute temperature. $k$ represents Boltzmann constant, $k=1.38 \times 10^{-23} \mathrm{~J} / \mathrm{K}$; the equivalent value of the input voltage noise of the amplifier at the output terminal is $\operatorname{Vin}=\left(1+\frac{R_{F}}{R i e}\right) \cdot e n \cdot \sqrt{B}$; the equivalent value of the input current noise of the amplifier at the output terminal is $V e n=R_{F} \cdot i n \cdot \sqrt{B}$; the thermal noise of the feedback resistor is $V_{R_{F}}=R_{F} \cdot \sqrt{\frac{4 k T B}{R_{F}}}$.

The sensitivity of the sensor is $S$, and the power of the incident light is $P$. After the signal of the photoelectric sensor passes through the detection circuit, the output voltage is

$$
V_{O}=I_{O} R_{F}=S P R_{F} .
$$

The SNR of the whole detection circuit is

$$
\begin{aligned}
\mathrm{SNR} & =\frac{V_{O}}{V n}=\frac{S P R_{F}}{\sqrt{B \cdot\left[R_{F}^{2} \cdot\left(\omega_{1}+\omega_{2}\right)\right]}} . \\
\omega_{1} & =\frac{4 k T}{R i e}+2 q \cdot\left(I_{O}+I_{D}\right)+i n^{2} \\
\omega_{2} & =4 k T R_{F}+\left(1+\frac{R_{F}}{R i e}\right)^{2} \cdot e n^{2}
\end{aligned}
$$

For a given amplifier, its parameters of $e n$ and in are fixed values, so the SNR of the whole detection circuit is mainly affected by $I o, I_{D}, R_{F}, B$.

\subsubsection{Bandwidth of the circuit}

From (11), we can see that the SNR and the bandwidth of the circuit have a great relationship. When all the electrical parameters $\left(e n, i n, I o, I_{D}, R_{F}\right)$ in the circuit are constant, (16) is established.

$$
\mathrm{SNR} \propto \frac{1}{\sqrt{B}}
$$

It shows that the bandwidth is not the bigger the better. However, with the raising of the SNR, the bandwidth will decrease, and the waveform of the laser pulse signal will be influenced. Accordingly, both SNR and the highfrequency component of the signal should be considered. Generally, the laser pulse is a Gaussian pulse, which is the quasi-rectangular pulse. While the bandwidth of the amplifier influences the response of the amplifier to the quasirectangular pulse [30].

If the $\Delta f \cdot \tau=0.5$ is met for the rectangular pulse, $(\Delta f$ is the $-3 \mathrm{~dB}$ bandwidth of the amplifier, $\tau$ is the rise time of the photoelectric conversion circuit), the ratio of the output pulse peak power and noise power is the largest, so the maximal SNR can be obtained [30].

The rise time of the 2-D photosensor is $1.5 \mu$ s. In order to match the performance of the detector reasonably, the $-3 \mathrm{~dB}$ bandwidth of the detection circuit should be around $330 \mathrm{KHz}$.

\subsubsection{Photocurrent and feedback resistance}

To analyze the influence of photocurrent $I_{O}$ on the SNR, the influence of dark current $I_{D}$ on the noise should be firstly considered. According to the principle of photoelectric detection, the dark current does not largely influence the SNR within the scope of possible changes. This is because the photocurrent is far greater than the dark current. However, when the photocurrent is weak (when the dark current and the photocurrent are comparable, such as in the presence of strong background light, the signal produced from the background light can be regarded as dark current), the influence of dark current will be aggravated. Thus the influence of the dark current cannot be ignored now.

When the situation of weak photocurrent is not considered, the dark current is regarded as a constant (maximum) $I_{D}=500 \mathrm{nA}$. Fig. 7 shows the relation of SNR with photocurrent $I o$ and feedback resistance $R_{F}$. It is observed that the photocurrent and resistance of feedback resistor all produce a large effect on the SNR from Fig. 7(a). Fig. 7(b) is the situation when azimuth is equal to 0 and elevation is equal to 0 in Fig. 7(a). It is observed that no matter how large the $R_{F}$ of the feedback resistor is, the photocurrent produces more influence on the SNR of the measuring 
circuit. Nevertheless, smaller $R_{F}$ values decrease this influence. Fig. 7(c) is the situation when azimuth is equal to 90 and elevation is equal to 0 in Fig. 7(a). Fig. 7(d) is the SNR curve with the changes of feedback resistance after the photocurrent value is discretized. It shows that when the photocurrent value is constant, increasing the value of $R_{F}$ of the feedback resistor properly can contribute to improving the signal-noise and raising the amplifier gain.

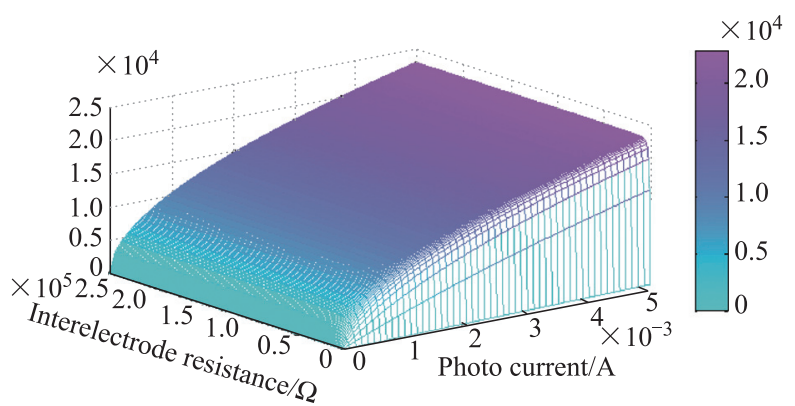

(a) Relationship between SNR, feedback resistance and photocurrent

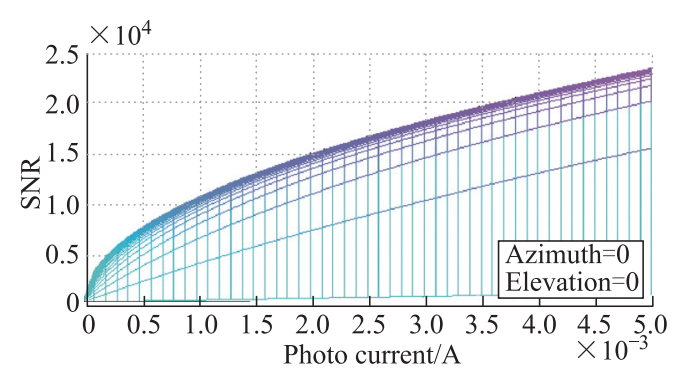

(b) Relationship between SNR and photocurrent

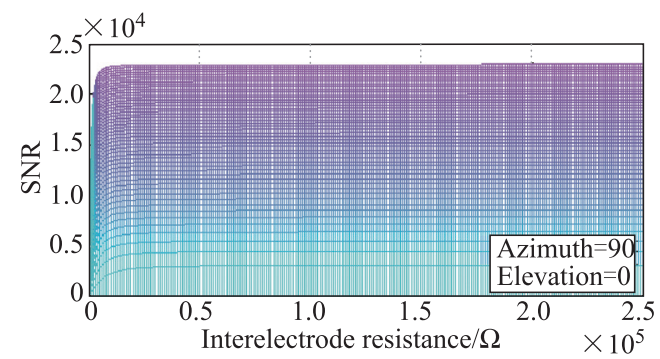

(c) Relationship between SNR and feedback resistance

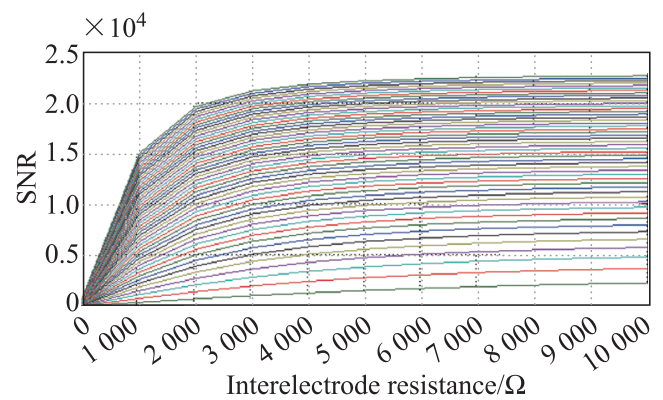

(d) Abscissa zoom of the relationship between SNR and feedback resistance

Fig. 7 Relationship between SNR, feedback resistance and photocurrent

\subsection{Design of detection circuit}

The above analysis and calculation show that an amplifier with low voltage noise under the high feedback is needed. To meet the above requirement, the trans-impedance amplifier (TIA) is adopted, while the decompensated op amplifier (DOA) is selected for higher bandwidth.

The stray capacitance $C_{S}$ that exists will form a zero with the feedback resistor $R_{F}$ on the gain curve $\left(Z_{1}\right.$ in Fig. 8), resulting in the approximation velocity at the intersection between the open-loop gain curve and noise gain curve of the operational amplifier approaching $-40 \mathrm{~dB} / \mathrm{dec}$. The result of intersection is that the instability of the operational amplifier will cause self-excited oscillation. Thus, to eliminate the self-excited oscillation of the circuit, usually a feedback capacitance $C_{F}$ is added for compensation, and a pole is introduced into the curve, with the compensated curve as shown in Fig. 8.

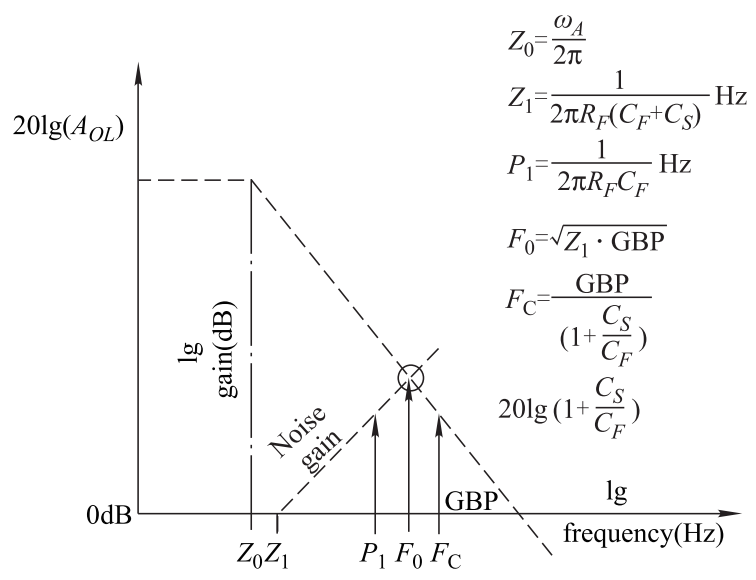

Fig. 8 Compensated Bode diagram

According to Fig. 8, to make the op amplifier to operate stably and reach the widest 2-order Butterworth frequency response, the value of feedback capacitance $C_{F}$ is set as follows:

$$
\frac{1}{C_{F}}=\sqrt{\frac{\pi \cdot G B P \cdot R_{F}}{C_{S}}} .
$$

When the target scattering distribution is the Lambert reflector, it is easy to calculate the photocurrent $I o$ of echo power [31] in the starting phase of terminal guidance to be 1.1e-05 A. The DOA adopts OPA657, whose electric parameters are $i n=1.3 \mathrm{f} \mathrm{A} / \mathrm{Hz}^{1 / 2}$, en $=4.8 \mathrm{n} \mathrm{V} / \mathrm{Hz}^{1 / 2}$. The change curve of noise and SNR with the change of feedback resistance $R_{F}$ is shown in Fig. 9 according to (4) and (6).

In the real application, although the SNR increases with the increase of feedback resistance, the fact is that not larger feedback resistance is better. On the one hand, the increase of feedback resistance will increase the noise of 
circuit. On the other hand, the increase of feedback resistance is difficult to guarantee the resistance of four channels of resistors matched to equal.

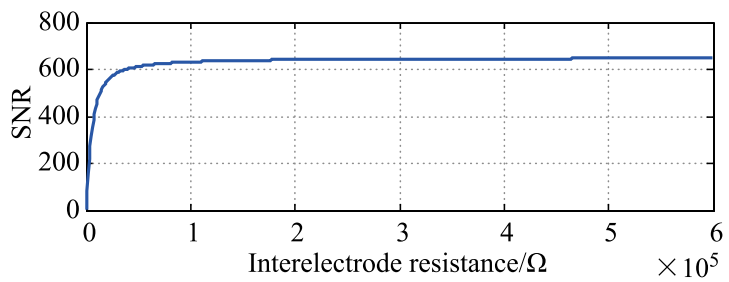

(a) SNR

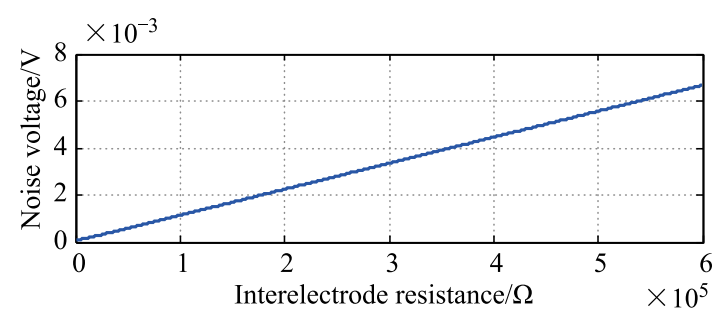

(b) Noise

Fig. 9 SNR and noise with feedback resistor

The resistor with high resistance has poor stability. Thus, the resistance of feedback $\left(R_{F}\right)$ resistor is set as $100-300 \mathrm{k} \Omega$.

According to the requirement on the correction precision, the SNR of circuit should be up to $53 \mathrm{~dB}$. Besides, the linear range of applicability of ADC devices on the rear end needs to be taken into consideration. And to prevent the system's dynamic range from being exceeded due to sudden increase of echo signal amplitude, the noise of circuit is $V$ Noise $=3.3 \mathrm{mV}$ and $\mathrm{SNR}=56 \mathrm{~dB}$ when the resistance of the feedback resistor is $300 \mathrm{k} \Omega$ in the maximum conditions. In this situation, the requirements on correction precision can be met.

According to the above analysis, the schematic diagram and parameters for the final detection circuit are made, as shown in Fig. 10. In the figure, $C 2$ is the junction capacitance of sensor, and $R 3$ and $C 3$ are the form of resistance capacitance (RC) filter circuit.

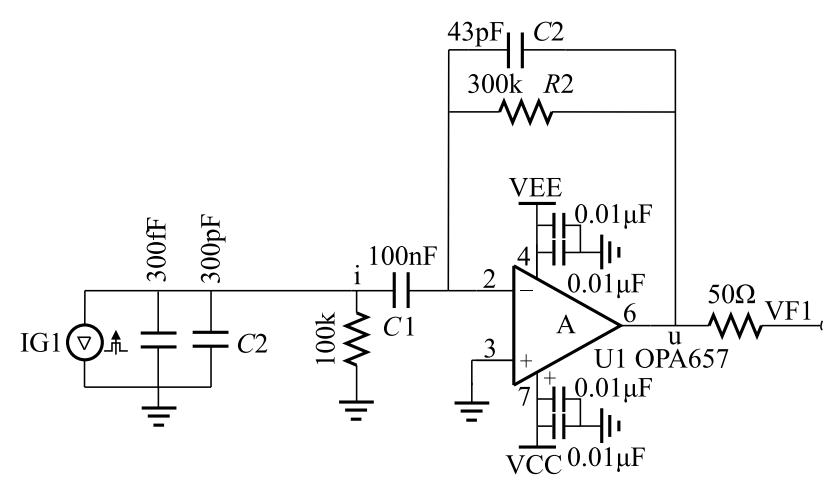

Fig. 10 Schematic diagram of detection circuit

The transfer function of signal extraction:

$$
\frac{U}{I}=-\frac{1}{\frac{R 1 \cdot C 1+R 2 \cdot C 2}{R 1 \cdot R 2 \cdot C 1}+\mathrm{j}\left(\frac{1}{R 1 \cdot R 2 \cdot C 1} \cdot \frac{w}{w_{0}}-C 2 \cdot \frac{w}{w_{0}}\right)}
$$

with

$$
w_{0}=\frac{1}{R 1 \cdot R 2 \cdot C 1 \cdot C 2} .
$$

Amplitude-frequency characteristics:

$$
F=-\frac{1}{\left(\frac{R 1 \cdot C 1+R 2 \cdot C 2}{R 1 \cdot R 2 \cdot C 1}\right)^{2}+\left(\frac{1}{R 1 \cdot R 2 \cdot C 1} \cdot \frac{w}{w_{0}}-C 2 \cdot \frac{w}{w_{0}}\right)^{2}}
$$

Phase-frequency characteristic:

$$
\phi_{F}=\tan ^{-1} \frac{R 0 \cdot \frac{w}{w_{0}}-C 2 \cdot \frac{w}{w_{0}}}{\frac{R 1 \cdot C 1+R 2 \cdot C 2}{R 1 \cdot R 2 \cdot C 1}}
$$

with

$$
R 0=\frac{1}{R 1 \cdot R 2 \cdot C 1}
$$

At resonance, the center frequency is

$$
f=\frac{1}{2 \pi \cdot \sqrt{R 1 \cdot R 2 \cdot C 1 \cdot C 2}} .
$$

When adapting the parametes, it is required to guarantee the frequency of the input coding laser light source is near the center frequency. What's more, the phase margin of the front end circuit should be considered to prevent the self-excited oscillation of circuit.

Then simulation is conducted using Texas Instruments (TI) spice-based analog simulation program (TINA) of TI, with result as shown in Fig. 11, which shows the circuit is stable and has no overshooting. The actual circuit is built, and the PSD output signal is connected to the sensor signal extraction circuit to measure the output waveform, as 
shown by the yellow line for channel one in Fig. 12 in details.

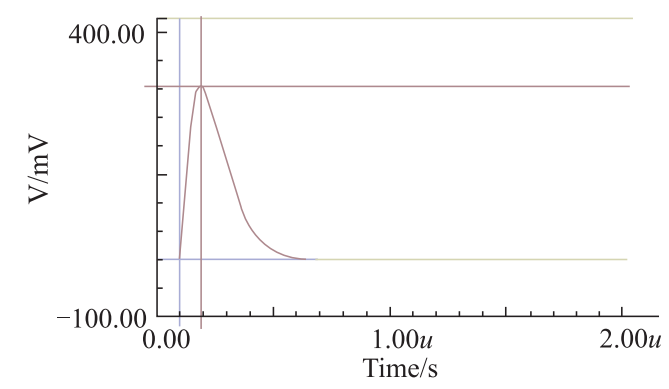

Fig. 11 Simulation results from TINA

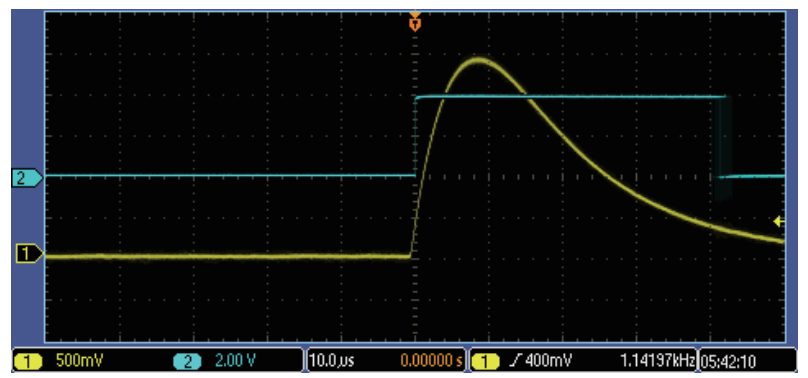

Fig. 12 The output waveform from actual circuit

\section{Experimental results and analysis}

To verify the proposed method, the laboratory experiment environment is built, shown in Fig. 13 in details.

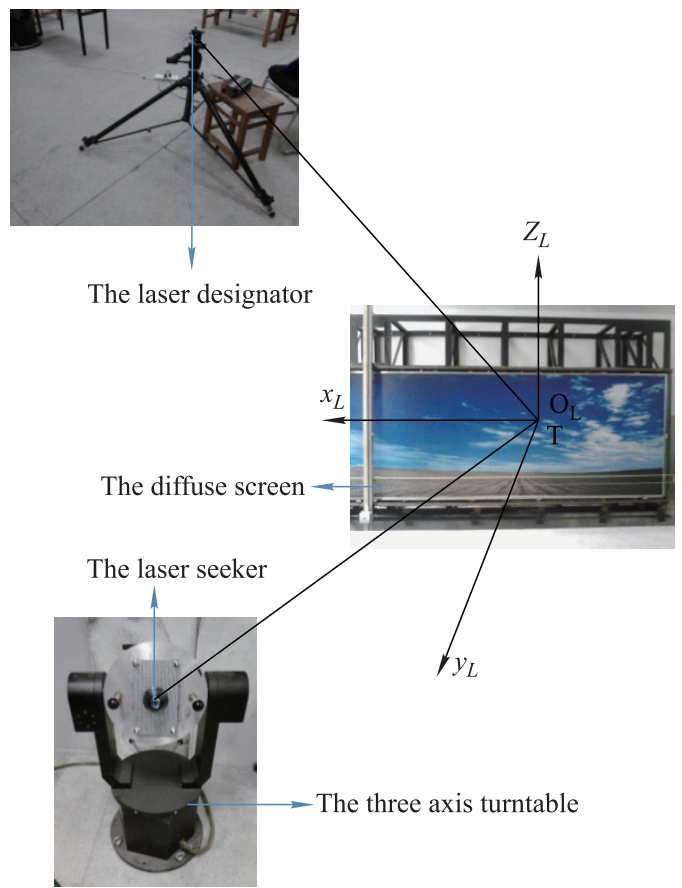

Fig. 13 Laser semi-active diffuse reflection experiment

The printed circuit board (PCB) was made according to the above analysis. In the laboratory, the laser was irradiated to the diffused reflection screen.
The inner axle, center axle and outer axle of the threeaxle table were adjusted to enable the seeker of the threeaxle table to receive the diffused reflection signal of the laser. The distance travelled by the laser beam was adjusted to make the photocurrent produced by the seeker about $0.01 \mathrm{~mA}$ and the data of the four channels was acquired using the digital acquisition card. The parameters of four channels are shown in Table 1.

Table 1 Experimental parameters table

\begin{tabular}{ccc}
\hline \multirow{2}{*}{ Channel } & \multicolumn{2}{c}{ Parameter } \\
\cline { 2 - 3 } & $\mathrm{CF} / \mathrm{pF}$ & $\mathrm{RF} / \mathrm{k} \Omega$ \\
\hline ONE & 2.2 & 20 \\
TWO & 7.5 & 50 \\
THREE & 43 & 300 \\
FOUR & 50 & 350 \\
\hline
\end{tabular}

Each power supply was turned on for data acquisition. The position of the laser was changed every time after data acquisition. The waveform of four channels is shown in Fig. 14.

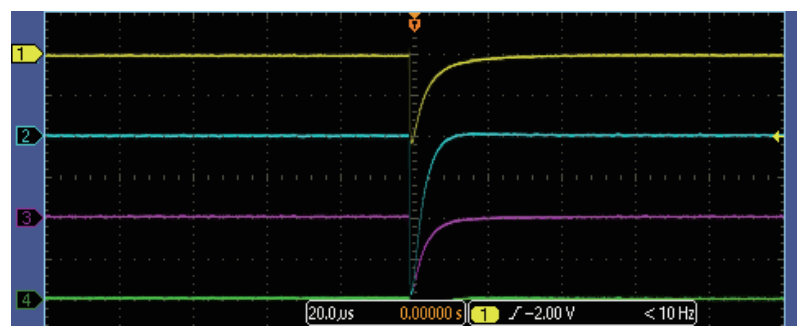

Fig. 14 Four wave signals of detection circuit

The data is subjected to fast Fourier transformation (FFT) processing in matlab. Firstly, the frequency represented by a certain point $n$ is calculated according to the result of processing: $F n=(n-1) \cdot F s / N . F s$ is the frequency of sampling; $N$ is the number of sampling sites. The first point represents the direct current (DC) component (i.e., $0 \mathrm{~Hz}$ ). The presence of the DC component is mainly caused by the environmental factors such as stray light and the noise in the system. Thus, during the process of calculating the coordinate of the spot point, the first step is that the module value of the first point is divided by $N$ and the DC component is obtained. Then according to the equation $n=F n \cdot N / F s+1$, the module value with the specific frequency is calculated, while the result should be divided by $N / 2$ to obtain the peak value of the original signal, with the result shown in Table 2.

In the process of data processing, the table only selects one channel of signals for processing each time, which can make the change trend of the noise easily seen. From the data of each channel, it can be seen that the trend of the influence of feedback resistance on the SNR ratio of the circuit and it is similar as shown in Fig. 9 in details. 
Table 2 Experimental results table

\begin{tabular}{ccccc}
\hline \multirow{2}{*}{ Times } & \multicolumn{4}{c}{ Noise/mV } \\
\cline { 2 - 5 } & $\begin{array}{c}\text { First } \\
\text { channel }\end{array}$ & $\begin{array}{c}\text { Second } \\
\text { channel }\end{array}$ & $\begin{array}{c}\text { Thrid } \\
\text { channel }\end{array}$ & $\begin{array}{c}\text { Fourth } \\
\text { channel }\end{array}$ \\
\hline 1 & 0.568 & 1.421 & 8.525 & 9.646 \\
2 & 1.572 & 2.443 & 9.512 & 10.623 \\
3 & 1.684 & 2.632 & 9.617 & 10.752 \\
4 & 2.243 & 3.250 & 10.248 & 11.567 \\
5 & 2.470 & 3.531 & 10.460 & 11.787 \\
\hline \multirow{3}{*}{ Times } & \multicolumn{4}{c}{ Peak value/V $($ SNR/dB) } \\
\cline { 2 - 5 } & First & Second & Thrid & Fourth \\
& channel & channel & channel & channel \\
\hline \multirow{2}{*}{1} & 0.185 & 0.694 & 4.312 & 4.890 \\
& $(50.2)$ & $(53.7)$ & $(54.1)$ & $(54.1)$ \\
2 & 0.503 & 1.178 & 4.752 & 4.997 \\
& $(50.1)$ & $(53.6)$ & $(53.9)$ & $(53.5)$ \\
3 & 0.519 & 1.224 & 4.664 & 4.997 \\
& $(49.7)$ & $(53.3)$ & $(53.7)$ & $(53.3)$ \\
4 & 0.628 & 1.430 & 4.733 & 4.997 \\
& $(48.9)$ & $(52.8)$ & $(53.3)$ & $(52.7)$ \\
\multirow{2}{*}{5} & 0.689 & 1.557 & 4.827 & 4.997 \\
& $(48.9)$ & $(52.8)$ & $(53.3)$ & $(52.7)$ \\
\hline
\end{tabular}

It is observed from Table 2 that with the increase of testing times, the noise takes on an uptrend, and is obviously larger than the theoretical value, while the SNR is decreased. The possible reasons are as follows: (i) with the increase of the working time of the circuit, the thermal noise and the temperature drift will influence the noise of the circuit; (ii) with the increase of the working time of the laser device, its efficiency will be lowered, and light intensity will be weakened; (iii) the influence of the background light will also increase the noise of the circuit; (iv) the layout of circuit and signal integrity (SI), etc. may also lead to increased noise. Despite of the increase of noise by several times, the SNR is not largely decreased. Paying attention to eliminating the stray light in designing the optical lens and solving the problems causing noise in designing the circuit can further reduce the noise and raise the SNR of the photoelectric detection circuit.

\section{Conclusions}

This paper firstly analyzes the imaging characteristics of the imaging face of the photosensor according to the trajectory information and the characteristics of correction projectile based on the laser seeker. Then the relation between the resolution of the rolling angle and the resolution of the spot point position is resolved. Finally, the detection circuit with the resolution errors of the rolling angle as the major parameter is designed. The experiment verification shows that this design can meet the requirement on the resolution error of the rolling angle in the semi-active laser seeker. In addition, for the laser seeker signal, the SNR of the circuit serves as the dominating influencing factor for the reso- lution error of the position. Actually, the fact is that not the larger the SNR is better. Therefore, by referring to the rolling angle resolution error and the transmission model of laser in the atmosphere, the satisfactory circuit can be easily designed. Considering the wide application areas of the photoelectric detection sensor in the semi-active laser weaponry in the future, the idea of this design can be taken as reference. Combined with the application of the photoelectric detection sensor in the related domains, its application value will be further improved.

\section{References}

[1] ZHANG Y, YANG R N, ZUO J L, et al. Tactic maneuver planning of loft delivery of laser-guided bomb with no offset. Systems Engineering and Electronics, 2016, 38(5): 1074-1080. (in Chinese)

[2] LI X L, YAO W J, ZHU L K, et al. Projectile roll angle measurement method of a combination of strapdown laser detector and GPS. Measurement for Semi-active Laser Terminal Correction, 2016, 2(13): $1000-1093$.

[3] WANG K B. Status quo, key technology and development of laser guided weapon. Infrared \& Laser Engineering, 2007, 36(5): 651-655. (in Chinese)

[4] AL-JABERI M, RICHARDSON M, COATH J, et al. The vulnerability of laser warning systems against guided weapons based on low power lasers. Journal of Battlefield Technology, 2006, 9(3): 9-12.

[5] ZHU Y, CUI X, WANG Q, et al. Analysis of laser energy characteristics of laser guided weapons based on the hardwarein-the-loop simulation system. Proc. of the SPIE/COS Photonics Asia Semiconductor Lasers and Applications VI, 2016: 1001715.

[6] LIANG W W, HUANG Z Y, ZHANG W P, et al. Study on error signal of quadrant detectors in laser seekers. Infrared Technology, 2014, 38(4): 569-573. (in Chinese)

[7] BURKLAND M K, STREUBER C T, TVEDT K E. Fixedsource array test station for calibration of a semi-active laser (SAL) seeker. US: US8392143, 2013.

[8] GAO Z, XU W. Design and analysis of displacement measurement system based on the four-quadrant detector. Proc. of the International Symposium on Photoelectronic Detection \& Imaging, 2013: 890531.

[9] LI X L, YAO W J, ZHU L K, et al. Ground target localization algorithm for semi-active laser terminal correction projectile. Defence Technology, 2016, 12(3): 234-241.

[10] GAO M, ZHANG Y, YANG S. Firing control optimization of impulse thrusters for trajectory correction projectiles. International Journal of Aerospace Engineering, 2015, DOI: $10.1155 / 2015 / 781472$.

[11] WANG Y, SONG W D, FANG D, et al. Guidance and control design for a class of spin-stabilized projectiles with a twodimensional trajectory correction fuze. International Journal of Aerospace Engineering, 2015, DOI: 10.1155/2015/908304.

[12] FAN S P, WU G, SUN Y, et al. Analysis of control coupling characteristic and forward decoupling technique for rolling missile. Systems Engineering and Electronics, 2017, 39(2): 398 - 403. (in Chinese)

[13] KARAPUZIKOV A I, PTASHNIK I V, SHERSTOV I V, et al. Modeling of helicopter-borne tunable TEA CO 2, DIAL system employment for detection of methane and ammonia leak- 
ages. Infrared Physics \& Technology, 2000, 41(2): 87-96.

[14] YU Y N. Research on weak current detection system of position sensitive detector. Wuhan, China: Wuhan University of Technology, 2012. (in Chinese)

[15] HENRY J, LIVINGSTONE J. Improved position sensitive detectors using high resistivity substrates. Journal of Physics D: Applied Physics, 2008, 41(16): 165106.

[16] SALVATORI S, MASARONE N, NUCCI G D, et al. Compact front-end electronics for low-level current sensor measurements. Electronics Letters, 2006, 42(12): 682-684.

[17] SORDO-IBÁÑEZ S, PIÑERO-GARCÍA B, MUÑOZ-DÍAZ $\mathrm{M}$, et al. CMOS rad-hard front-end electronics for precise sensors measurements. IEEE Trans. on Nuclear Science, 2016, 63(4): $2379-2389$.

[18] LI C Y, CHENG Z, CHEN F, et al. Design of a photoelectric detection circuit in particle analysis apparatus for clinical liquid sample. Applied Mechanics \& Materials, 2015, 696: 134140.

[19] ZHAN J M, WEN D S, WANG H, et al. Noise analysis of pre-amplifier based on photodiode. Semiconductor Technology, 2011, 36(4): $304-299$. (in Chinese)

[20] CAO Y J, ZHANG E D, CUI Q, et al. Design of a high precision integrated charge detection circuit based on quartz gyroscope. Advanced Materials Research, 2011, 317-319: 1143 1148.

[21] FANG R, WANG C. Design and analysis of APD photoelectric detecting circuit. Proceedings of SPIE, 2015: 9795.

[22] ZHOU Y J, REN K, QIAN W X, et al. Noise analysis of photoelectric detection circuit based on photodiode reverse bias. Infrared and Laster Engineering, 2016, 45(1): 252-257.

[23] RYDYGIER P, FIUTOWSKI T, DABROWSKI W. Design of a low noise, low power, high dynamic range amplifier-filter circuit for recording neural signals using multielectrode arrays. Proc. of the IEEE Mixed Design of Integrated Circuits \& Systems, 2010: $242-247$.

[24] KIM D, GOLDSTEIN B, TANG W, et al. Noise analysis and performance comparison of low current measurement systems for biomedical applications. IEEE Trans. on Biomedical Circuits \& Systems, 2013, 7(1): 52-62.

[25] JIANG X G, LI H, YANG X L, et al. Noise analysis of the measurement circuit for position sensitive detector. Information \& Electronic Engineering, 2010, 8(1): 96-100.

[26] MIN R, XU Y, SUN Z. Fundamentals of electronic circuits. Xi'an: Xidian University Press, 2003: 180-220. (in Chinese)

[27] BEIKAHMADI M, MIRABBASI S. A low-power low-noise CMOS charge-sensitive amplifier for capacitive detectors. Proc. of the IEEE New Circuits and Systems Conference, 2011: 450-453.

[28] BO W, WEI A N, KAI X, et al. Real-time sensor scheduling algorithm in LEO constellation based on pruning. Systems En- gineering and Electronics, 2010, 32(6): $1244-1250$. (in Chinese)

[29] WU X. The technology on noise reduction of the APD detection circuit. Proc. of the International Symposium on Photoelectronic Detection \& Imaging, 2013: 890525.

[30] BO G Y, QI J, LI J, et al. Design method of preamplifier circuit with THS4012 in laser altimeter. Journal of Atmospheric and Environmental Optics, 2007, 2(4): 316-320.

[31] KLEIN L A. Millimeter-wave and infrared multisensor design and signal processing. Norwood, MA: Artech House Publishers, 2002.

\section{Biographies}

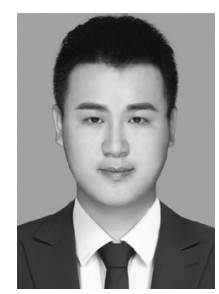

ZHU Likun was born in 1991. He is a lecturer in Nanjing University of Science and Technology. His research interests are digital signal processing, parallel heterogeneous computing, weapon guidance and so on.

E-mail: aaa123@yahoo.cn

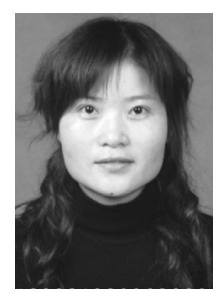

JIA Fangxiu was born in 1980. She is a Ph.D. and a lecturer in Harbin Institute of Technology. Her research interests are design of micro-electromechanical system inertial sensor circuit and inertial measurement unit attitude measurement technology. E-mail: jiafangxiu@gmail.com

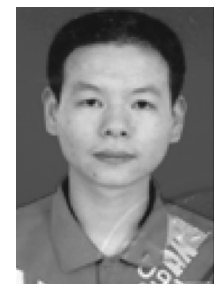

JIANG Xiaodong was born in 1994. He is a lecturer in Nanjing University of Science and Technology. His research interest is intelligent ammunition technology.

E-mail: jxd0204@126.com

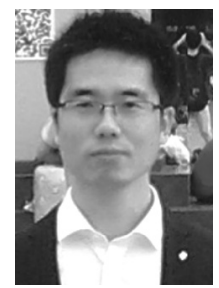

LI Xinglong was born in 1988. He is a Ph.D. and a lecturer in Nanjing University of Science and Technology. His research interest is intelligent ammunition technology. At present, he works in the Institute of Chemical Materials, China Academy of Engineering Physics.

E-mail: lixinglong.sj@163.com 